\title{
Editorials
}

\section{THE PEOPLE WE FAIL TO REACH}

Leprosy is a disease which affects the body of the patient and the mind of the public. It carries a greater stigma than any other disease and the continued use of the word "leper" with its evil connotation is still commonly used in most societies. Can we truly blame the public when the majority of the medical profession continue to treat it as a disease apart from all others?

The age-old fear of leprosy was the result of the unsightly deformities and the fact that no cure was available. Today leprosy is curable, deformities can be prevented and if they should occur can be corrected by reconstructive surgery.

Nevertheless the age-old fear and stigma continues to persist and is probably the single most important factor which has prevented the control of leprosy, despite well planned national programmes based on effective drugs and modern scientific knowledge. Despite the expenditure of vast sums of money we realize that only a third of the estimated number of persons in the world suffering from leprosy are actually registered, and surveys demonstrate that of these less than $50 \%$ take regular if any treatment after diagnosis. At the end of 2 years probably another half drop out or become irregular. How can any disease be controlled, however effective the drugs, if the majority of patients do not take the treatment necessary for the cure? This is a problem common to many other chronic diseases like tuberculosis and filariasis.

Unfortunately these important social and psychological aspects of the disease receive scant attention in the planning of most major programmes, and the emphasis continues to be on "early detection and treatment". It has been demonstrated by the Belgian Centre at Polambakam that the addition of physiotherapy and surgery considerably improved regularity of attendance, for the patient felt something more was being done than mere distribution of pills. The experience of the Danish programme in Pogiri and Aksa was even more remarkable in that by education and ready availability of treatment, not only did the majority of patients come voluntarily for treatment but also that the regularity rate was much higher because the patient realized the importance of such treatment. It is also interesting to note that this programme had a minimal medical staff component.

These and other similar experiences have demonstrated that social and psychological factors are probably more important in leprosy control than the medical component of the programme. While it is not suggested that the medical aspect of the disease is unimportant, the medical profession who advise governments and plan most programmes have failed to realize the relative importance of the above factors which are hence generally given only token recognition, as is reflected in most budgets for leprosy control.

There are many booklets, posters, slides and other educational materials which 
have been made available for leprosy in the last 2 decades. Public lectures are given, as well as radio talks and press publicity. Why is it then that the public attitude towards the disease has not shown any significant change? Unfortunately much of this educational effort, though well intentioned, is ill conceived and poorly executed. The appeal is often emotional and based on pathos rather than directed towards a more intellectual approach commensurate with modern scientific knowledge about this disease. Sometimes this is excused on the plea that only thus can funds be raised. Why is it that a grossly deformed victim is the usual picture rather than a cheerful young girl with a small skin patch? The latter is certainly more true to reality. The more factual presentations are usually restricted to small meetings, where the preacher preaches to an audience already converted, or through posters in leprosy institutions.

A change in public attitude can be achieved only by a sustained campaign of education using mass media of publicity. The radio, television and the press are potent but not the only available media. The schools, political platforms, and word of mouth are also important tools in the art of communication, education and persuasion. All these techniques are being used daily whether for the sale of soap or matches, for the sale of drugs by pharmaceutical firms or ideologies by politicians, and they reach the remotest village. That these methods can be employed in the field of health has been demonstrated by the campaigns for family planning and smallpox and poliomyelitis eradication.

In order to test this hypothesis 2 programmes were conducted in Bombay. One was an essay competition in about 50 schools, with teacher and student participation. About 100,000 students took part. Material on leprosy was provided for the teacher and after a suitable period the essays were evaluated and prizes distributed to the students, teachers and the schools at a public ceremony presided over by a popular dignitary, and hence well publicized in the press. This has been carried out over the past several years but since the programme was not varied the earlier enthusiasm has waned. The extent of knowledge of the disease was evaluated by a questionnaire filled by each student, and indicated a high level of awareness of the disease and its acceptance by a receptive group of the community.

The second experiment consisted of a weekly 10 minute radio broadcast in English by a well known former cricketer and radio commentator. This was continued over a period of about 2 years. At the end of the cricket anecdotes a couple of minutes were devoted to giving simple facts on leprosy. Though the English medium greatly restricted the audience, the response as measured by the mail was encouraging. The above 2 experiments conducted by a private organization in Bombay only indicate the possibility of the use of media for mass education. The result of such education is not easy to perceive in the short term, but methods of evaluation are available though expensive.

Our limited experience in leprosy and the vast experience of the effectiveness of mass media of communication in other fields indicates the need for the use of such an approach if we are to change public opinion and attitudes. Such education can be carried out most effectively by the respective governments as a part of their national leprosy control programme using the available expertise in this field, and with the technical guidance being provided by the medical profession.

A major handicap in the control of leprosy has been the unscientific attitude of the medical profession. This has resulted in the formation of a vertical programme 
for leprosy. While vertical programmes may have certain advantages in the control of diseases like malaria and smallpox, they have proved a handicap when dealing with diseases like tuberculosis, venereal disease and leprosy. Such programmes perpetuate the stigma in the minds of the public and the medical profession. The latter is demonstrated by the failure to attract suitable personnel and the attachment of the stigma to those who join. If figures were available of the medical posts lying vacant in the national leprosy services as well as of the quality and turnover rate of those that are filled, they would reveal an important reason for the failure of our programmes. Fortunately the younger members of the medical profession do not suffer from the intense fear which prevailed among the older generation.

Our experience in the past 17 years in a large medical school has revealed that medical students, nurses and other personnel are willing to accept leprosy as any other disease if it is taught to them as part of the routine medical curriculum, and if they can see leprosy patients in the general outpatients and wards. Segregation and barrier nursing of infectious cases should be practised as in any other contagious disease. No patient has ever complained of leprosy cases being treated in our general ward.

It is my belief that the integration of leprosy in general medical education and hospital practice would probably prove a most potent factor in overcoming the stigma not only among the medical, nursing and paramedical workers but also among the lay public. A change in medical education is thus a prerequisite to integration and removal of stigma.

The recent emphasis on the delivery of health care to the rural and the poorer sections of the urban population by the training of paramedical workers from the community provides an opportunity to reach out to a large section of the population who are not adequately covered by the present leprosy services. The inclusion of simple facts about leprosy in the training of such workers will not only help in early detection but may also ensure improvement in regularity of treatment.

Lack of appreciation of the significance of social and psychological factors has resulted in the failure of otherwise well conceived programmes for the treatment and control of many diseases. Even where a "one shot" cure is available as in syphilis and gonorrhoea, control has not been achieved even in affluent countries. In a disease like leprosy where social stigma is even greater and where treatment has to be taken regularly over the years, programmes based chiefly on the medical concept are doomed to failure. It is time that detailed studies be undertaken by social scientists to delineate the factors responsible for the failure of our present control programmes. Based on the information : from such studies a more realistic approach may be devised and the available budget be utilized to greater advantage.

N. H. ANTIA 